${ }^{1}$ Facultad de Medicina, Universidad de los Andes, Santiago, Chile.

${ }^{2}$ Unidad de Estudios Cardiológicos, Clínica Santa María, Santiago, Chile. ${ }^{3}$ Departamento de Enfermedades Cardiovasculares, Clínica Dávila, Santiago, Chile.

${ }^{4}$ Departamento de Cardiología, Hospital San Juan de Dios, Santiago, Chile.

aBioquímico, Doctor en Ciencias Biológicas.

${ }^{\mathrm{b}} \mathrm{PhD}$ in Health and Biology.

Fuente de apoyo financiero: Ninguna.

Recibido el 19 de agosto de 2013, aceptado el 21 de julio de 2014.

Correspondencia: Dr. Jorge Bartolucci Johnston Unidad de estudios cardiológico, Clínica Santa María. Fernando Manterola 0530, Providencia.

Teléfonos: 9132958-9132959. jbartolucci@csm.cl

\section{Estado actual de la terapia con células madre en el tratamiento de las cardiopatías}

\author{
JORGE BARTOLUCCI ${ }^{1,2}$, FERNANDO J. VERDUGO ${ }^{1}$, \\ RICARDO LARREA ${ }^{1,3}$, FLAVIO CARRIÓN ${ }^{1, a}$, RUBÉN LAMICH ${ }^{2}$, \\ PABLO PEDREROS ${ }^{2}$, MANUEL DELGADO ${ }^{2}$, PATRICIO SANHUEZA ${ }^{4}$, \\ MAROUN KHOURY ${ }^{1, b}$, FERNANDO E. FIGUEROA ${ }^{1}$
}

\section{Stem cells for the treatment of cardiovascular diseases. An update}

Available medical therapy is unable to completely prevent or revert the pathological cardiac remodeling secondary to ischemia or other injuries, which is responsible for the development of heart failure. Regenerative medicine through stem cells had an explosive development in the cardiovascular area during the past decade. Stem cells possess the capacity to regenerate, repair or substitute damaged tissue, allowing the reestablishment of its function. Stem cells can also modulate apoptosis, angiogenesis, fibrosis and inflammation, favoring the endogenous regenerative process initiated by the damaged tissue. These capacities have been corroborated in several animal models of cardiovascular diseases with positive results. In humans, therapies with bone marrow mononuclear stem cells, mesenchymal stem cells and cardiac stem cells are safe. Most randomized clinical trials in patients with myocardial infarction or cardiomyopathies of different etiologies have reported benefits on ventricular function, quality of life and even over mortality of treated patients. This article reviews the state of art of stem cell therapy in cardiovascular diseases, focusing on the most common cellular types used in patients with acute myocardial infarction and chronic cardiomyopathies of different etiologies.

(Rev Med Chile 2014; 142: 1034-1046)

Key words: Cardiomyopathies; Myocardial Infaction; Stem Cell Transplantation; Ventricular Remodeling.
L as enfermedades cardiovasculares representan la primera causa de muerte en el mundo por sobre el cáncer y las enfermedades infecciosas $^{1}$. Su incidencia ha presentado un significativo incremento, incluso a edades tempranas, fenómeno asociado a la mayor prevalencia de factores de riesgo cardiovascular ${ }^{2}$. A pesar del enorme progreso de la terapia farmacológica, la masificación de la angioplastía primaria y la incorporación de nuevos dispositivos, las cuales tienen un positivo efecto sobre el pronóstico y la calidad de vida ampliamente reportada; las tasas de hospitalización y mortalidad cardiovascular permanecen elevadas y representan considerables costos de salud ${ }^{3}$. El arsenal terapéutico disponible no ha logrado revertir en su totalidad los cambios maladaptativos del miocardio, producto de la isquemia u otras injurias, lo cual conduce a un remodelamiento patológico del miocardio, pérdida progresiva de la función contráctil y el desarrollo de insuficiencia cardiaca. Estos importantes antecedentes epidemiológicos y clínicos han impulsado el desarrollo de nuevas formas de tratamiento de las enfermedades cardiovasculares y sus complicaciones. 
La medicina regenerativa mediante el uso de células madre o progenitoras ha tenido un rápido desarrollo en el ámbito de la investigación cardiovascular. Estas células poseen la capacidad de diferenciarse hacia otros tipos celulares maduros, sugiriendo la posibilidad de regenerar, reparar o sustituir el tejido dañado con el objetivo de restaurar su función ${ }^{4}$. La posibilidad de favorecer la regeneración miocárdica mediante la administración de células del mismo paciente (autólogas) u otro individuo (alogénicas) representa una interesante oportunidad para reemplazar el tejido miocárdico necrótico y favorecer la limitada reparación endógena del corazón. No extraña entonces que pasaran pocos meses entre la publicación del primer trabajo que demostró regeneración miocárdica en ratones infartados, tratados con células de médula ósea, y el inicio de una serie de ensayos clínicos no controlados, en pacientes con infarto agudo al miocardio (IAM) ${ }^{5-8}$. Los resultados de estos primeros ensayos han generado un creciente desarrollo de investigación experimental, con rápida traslación hacia investigaciones clínicas de mayor calidad. A continuación revisaremos el estado del arte del tratamiento con células progenitoras de patologías cardiovasculares, enfocándonos en los tipos celulares más utilizados, su aplicación en el IAM y en cardiopatías crónicas de diferente etiología.

\section{Tipos y fuentes de células madre en cardiología}

Las células madre son células caracterizadas por su capacidad de auto-renovación (dividirse en más células madre de forma indefinida) y el potencial de diferenciarse en células maduras de un determinado linaje celular. En términos amplios, podemos clasificar las células madre según su potencialidad en células madre pluripotenciales (embrionarias o inducidas) y multipotenciales (adultas). Un creciente volumen de evidencia preclínica, avala el gran potencial terapéutico de las células madre pluripotenciales inducidas (iPSC) en patología cardiovascular-11. Existen sin embargo, dudas razonables respecto a su eventual efecto tumorigénico y/o inmunogénico, lo que ha dificultado por ahora su traslado a la clínica ${ }^{12,13}$. Considerando su aplicación clínica, haremos referencia a las células madre multipotenciales.
Las células madre multipotenciales son capaces de diferenciarse a un espectro más limitado de tipos celulares maduros, dentro de un mismo linaje germinal ${ }^{13}$. Se encuentran en prácticamente todo órgano de los organismos multicelulares, independiente de su tasa de recambio celular en condiciones fisiológicas, confinadas en microambientes o nichos que regulan su actividad ${ }^{4,13}$. Si bien se presume que las células multipotenciales tendrían función y diferenciación tejido específica, evidencias recientes sugieren que bajo ciertas condiciones pueden reprogramarse y diferenciarse a células de otros tejidos, capacidad conocida como plasticidad o transdiferenciación celular ${ }^{13,14}$. Esto permite obtener células madre de fuentes con una mayor cantidad de células madre y/o más fácilmente asequibles. Adicionalmente, estas células pueden ejercer un efecto paracrino mediante la secreción de citoquinas y factores de crecimiento. Las células madre más estudiadas en cardiología corresponden a las células mononucleares de medula ósea (BMMC), células madre mesenquimales (MSC) y células madre cardiacas (CSC).

Las BMMC comprenden una serie de subpoblaciones de células madre y progenitoras, incluyendo células madre hematopoyéticas y en menor proporción MSC. Poseen una serie de ventajas para la terapia celular, son de fácil acceso mediante aspiración medular, aislables y expandibles en cultivo celular.

Las MSC han sido aisladas en diversos tejidos adultos (médula ósea, tejido adiposo, cordón umbilical, placenta), presentando diferentes morfologías, capacidades de diferenciación y expresión génica según la fuente de origen ${ }^{15,16}$. Dada su baja inmunogenicidad, las MSC de jóvenes sanos o cordón umbilical pueden ser implantadas en pacientes de edad avanzada o con patologías crónicas. Esta propiedad alogénica, resulta particularmente útil considerando que la efectividad de la terapia celular depende de la capacidad proliferativa de las células administradas, capacidad afectada negativamente por la edad, los factores de riesgo cardiovascular y patologías crónicas (insuficiencia cardiaca y diabetes mellitus entre otras) ${ }^{17}$.

La identificación de CSC como células endógenas del miocardio, rompe un paradigma de la fisiopatología cardiovascular, al demostrar que el corazón no sería un órgano post mitótico incapaz de auto-renovarse ${ }^{18}$. Es posible aislar y expandir CSC desde biopsias miocárdicas realizada median- 
te un procedimiento mínimamente invasivo o quirúrgico. Las CSC tendrían un mayor compromiso de diferenciación hacia cardiomiocitos, células musculares lisas y células endoteliales, manteniendo un importante componente paracrino ${ }^{18-22}$. Aún es motivo de investigación si subpoblaciones de estas células (diferenciadas por marcadores moleculares) poseen distintos efectos terapéuticos ${ }^{20}$.

\section{Mecanismos de acción de células madre en el corazón patológico}

Dependiendo de su potencialidad, estudios in vitro e in vivo han demostrado la capacidad de las células madre de transdiferenciarse en células similares a cardiomiocitos, capaces de formar sarcómeros y expresar marcadores moleculares de estos (miosina, actinina alfa, troponina, conexina-43 $)^{5,23,24}$. La capacidad de transdiferenciarse en células endoteliales y músculo liso vascular, explicaría parcialmente el aumento en la densidad capilar, así como en el aumento de vasos colaterales descritos en modelos animales de IAM y miocardiopatía dilatada sometidos a terapia celular ${ }^{5,23-25}$. Como mecanismo alternativo a la transdiferenciación, algunos autores han plan- teado una fusión de las células infundidas con cardiomiocitos residentes ${ }^{26,27}$.

Las células madre ejercen además un importante efecto paracrino, sintetizando y secretando una amplia variedad de factores de crecimiento y citoquinas con efectos antiapoptóticos (bFGF, IGF-1), proangiogénicos (VEGF) y antifibróticos (TNF $\alpha$, metaloproteinasas) $)^{6,16,22,28}$. El efecto de estas citoquinas explicaría parcialmente la inhibición de apoptosis y fibrosis, la neovascularización y la mejoría del remodelamiento ventricular, reportado en modelos experimentales ${ }^{24,25,29}$. Otros autores sugieren que mediante la secreción de citoquinas, las células madre exógenas pueden inducir la activación, migración y diferenciación de células madre cardiacas intrínsecas o estimular la proliferación de cardiomiocitos residentes ${ }^{28,30}$. Las células madre han demostrado un considerable efecto inmunorregulatorio, pudiendo inhibir la producción de citoquinas proinflamatorias (TNF- $\alpha$, IL-1, IL-6) y estimular la expresión de otras antiinflamatorias (IL-10) ${ }^{31,32}$. Esto cobra relevancia para pacientes cardiópatas, pues niveles plasmáticos elevados de TNF- $\alpha$ e IL- 6 se asocian a mayor daño miocárdico ${ }^{33,34}$.

Los mecanismos antes descritos se resumen en la Figura 1.

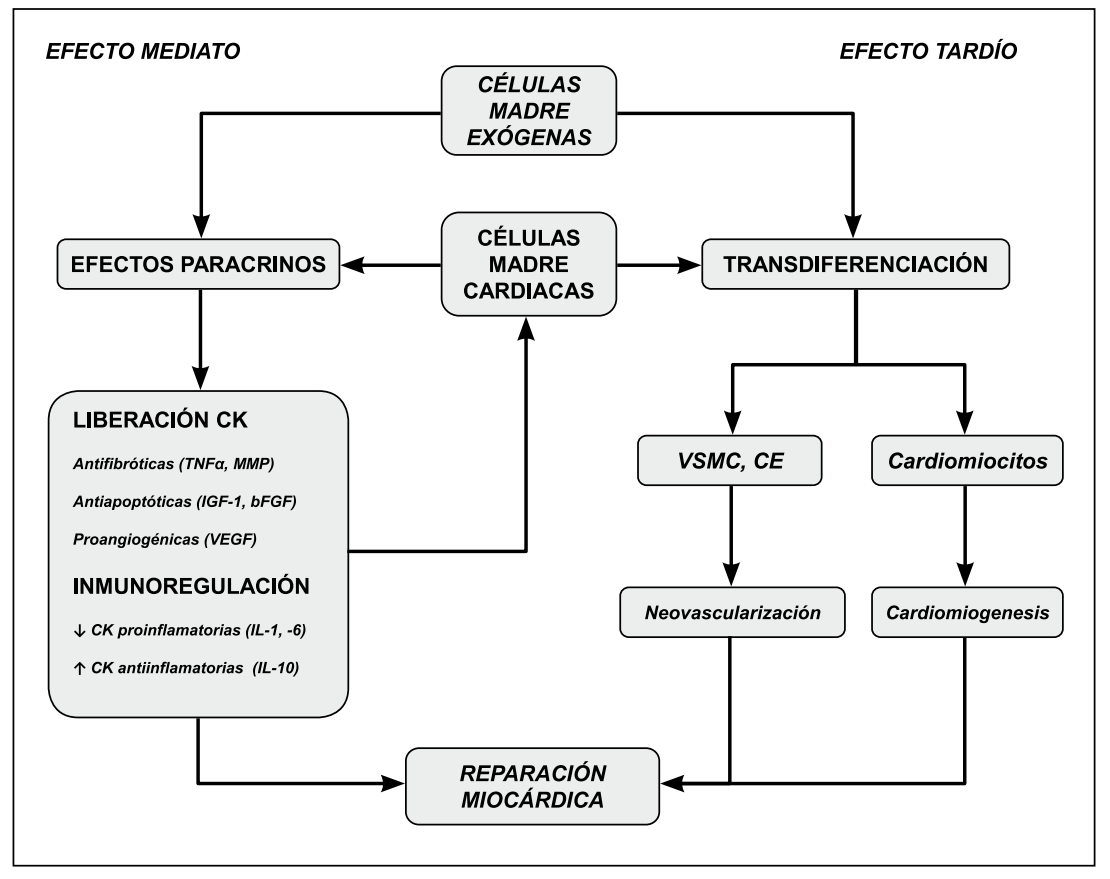

Figura 1. Mecanismos de acción terapéutica de las células madre. Se exponen de forma simplificada los principales mecanismo por los que la terapia celular contribuye a la reparación miocárdica en infarto agudo al miocardio y en cardiopatías crónicas de diversa etiología. CK: Citoquinas. TNF $\alpha$ : Factor de necrosis tumoral alfa. IGF1: Factor de crecimiento insulínico tipo 1. bFGF: Factor de crecimiento fibroblastico básico. VEGF: Factor de crecimiento endotelial vascular. VSMC: Células de músculo liso vascular. CE: Células endoteliales. 
Terapia con células madre en cardiopatías - J. Bartolucci et al

\section{Vías de implante}

Las vías de implante más frecuentemente utilizada en ensayos clínicos ha sido la infusión intracoronaria vía catéter con balón, procedimiento considerado seguro, sin embargo, existen reportes de una mayor tasa de reestenosis intrastent en pacientes pretratados con factores estimulantes de colonias de granulocitos ${ }^{35}$. La inyección intramiocárdica mediante catéter endoventricular o durante cirugía cardiaca, permite una administración dirigida hacia el área afectada, con el objetivo de aumentar la eficacia de la terapia celular, sin causar obstrucción vascular, reestenosis ni formación de ateroma. Es técnicamente más compleja, costosa y conlleva el riesgo de perforación miocárdica ${ }^{14}$. Los meta-análisis no demuestran preferencia por una u otra vía de administración en cardiópatas ${ }^{36}$.

La administración por vía venosa periférica es una estrategia menos invasiva, simple y segura que permite la infusión una mayor cantidad de células, todas las veces que se requiera. Algunos autores sostienen que esta vía puede ser tan efectiva como la infusión intracoronaria en pacientes infartados reperfundidos ${ }^{51}$. Nuestro grupo está desarrollando el ensayo aleatorio RIMECARD, cuyo objetivo principal es estudiar la seguridad y eficacia terapéutica, de la administración endovenosa de MSC de origen placentario, en pacientes con cardiopatía dilatada de diferente etiología (Número de registro en Clinicaltrials.gov NCT01739777).

\section{Terapia celular en infarto agudo al miocardio}

A la fecha, numerosos estudios experimentales han señalado que el trasplante de células madre autólogas o alogénicas, es capaz de mejorar la fracción de eyección del ventrículo izquierdo (FEVI), disminuir el tamaño del infarto y prevenir el remodelamiento ventricular patológico ${ }^{5,6,23,29,37}$.

En el ámbito clínico, el impacto de terapias celulares con BMMC sobre la función ventricular y la prevención del remodelamiento patológico han sido evaluadas por más de 50 estudios clínicos entre estudios de cohorte y ensayos clínicos fase $1-2^{7,8,38-53}$. Entre los estudios con BMMC autólogas de médula ósea, destaca el estudio BALANCE, cohorte con seguimiento a 60 meses que ha concluido que el trasplante intracoronario de BMMC, conduce a una mejoría significativa y prolongada de la función ventricular, mejorando también la calidad de vida y la mortalidad post IAM $^{46}$. Estos resultados han sido corroborados por los estudios TOPCARE-AMI y STIM, que señalan una mejoría persistente de la FEVI y una reducción significativa del tamaño de infarto ${ }^{40,50}$. Por otro lado, el estudio BOOST reportó mejorías significativas de la FEVI a 6 meses de la infusión intracoronaria de BMMC en pacientes infartados, aunque no demostró ningún efecto persistente sobre este parámetro a 18 y 60 meses de seguimiento ${ }^{53-55}$.

Un meta-análisis reciente comprendiendo 50 estudios randomizados $(n=2625)$, ha reportado que pacientes tratados con BMMC presentarían una disminución de la mortalidad general (OR $0,39 ; \mathrm{p}<0,00001)$ y del riesgo de infarto recurrente $(\text { OR } 0,25 ; p=0,001)^{36}$. La terapia celular también se asoció a una mejoría significativa de la FEVI $(+3,96 \% ; \mathrm{p}<0,00001)$, menor tamaño de infarto $(-4,03 \% ; \mathrm{p}<0,00001)$, menor volumen de fin de sístole $(-8.91 \mathrm{ml}, \mathrm{p}<0,00001)$, y menor volumen de fin de diástole $(-5,23 \mathrm{ml} ; \mathrm{p}<0,0001)^{36}$. Estos datos han sido interpretados recientemente por expertos en IAM, como un claro indicio de la efectividad clínica de la terapia celular, estimulando la búsqueda de nuevas estrategias y productos celulares. Un hecho importante y coincidente entre todos los estudios previamente citados, es que no se han observado efectos secundarios a la terapia celular.

Algunos de los efectos beneficiosos de la infusión de BMMC sugieren ser dosis dependientes. Los meta-análisis indican mejorías evidentes de la FEVI, tamaño de infarto y volúmenes ventriculares en pacientes que recibieron $\geq 40 \times 10^{6} \mathrm{BMMC}$ versus quienes recibieron $<40 \times 10^{6} \mathrm{BMMC}$, en quienes la mejoría de alguno de estos parámetros esta menos descrita ${ }^{36}$. Estudios controlados que han comparado el efecto de dosis bajas contra altas de BMMC por vía intracoronaria, han documentado que estas últimas se correlacionan con una mejoría de la función miocárdica regional, de la perfusión miocárdica y una mayor reducción del tamaño de infarto, sin asociarse a mayores complicaciones ${ }^{51,52}$.

El efecto terapéutico de las MSC también ha sido explorado en pacientes con $\mathrm{IAM}^{38,56-58}$. La mayoría de estos estudios han empleado células autólogas, menos regenerativas en el caso de los pacientes cardiópatas, infundidas por vía intracoronaria o intramiocárdica. Probablemente el trabajo más interesante desde el punto de vista biológico y clínico con MSC, sea el estudio PRO- 
CHYMAL $^{58}$. Se trata del primer empleo de MSC alogénicas en patología cardiovascular humana, aplicadas en un ensayo clínico aleatorizado, doble ciego, controlado con placebo y con escalamiento de dosis hasta $5 \times 10^{6} \mathrm{MSC} / \mathrm{kg}$, con células expandidas de sólo un donante y administradas por vía venosa periférica, dentro de la primera semana post $\mathrm{IAM}^{58}$. Los pacientes tratados, presentaron a los 6 meses de seguimiento: una menor incidencia de taquicardia ventricular, mejoría de las pruebas de función pulmonar y aumento de la FEVI, asociada a una reversión del remodelamiento miocárdico $^{58}$. Considerando los resultados auspiciosos, se encuentra en desarrollo una segunda etapa del ensayo que considerará 220 pacientes adicionales (NCT00877903).

La investigación clínica actual con CSC se ha enfocado en determinar la seguridad de la administración de CSC primitivas o cardioesferas, que resultan de la combinación combinación de CSC primitivas con otras más diferenciadas ${ }^{20}$. El ensayo clínico aleatorizado SCIPIO $(n=23)$ realizado en pacientes con cardiopatía coronaria y disfunción sistólica, revascularizados mediante bypass coronario, reportó un aumento significativo de la FEVI y disminución del tamaño de infarto en los pacientes infundidos con CSC primitivas por vía intracoronaria ${ }^{59}$. El estudio CADUCEUS (n =25) demostró una disminución del tamaño de infarto y aumento de la motilidad regional tras la infusión intracoronaria de cardioesferas luego de 1,5-3 meses post IAM con disfunción sistólica ${ }^{60}$. No encontraron cambios de la FEVI ni volúmenes ventriculares ${ }^{60}$. Ambos ensayos clínicos sugieren la seguridad del uso terapéutico de CSC luego de 1 año de seguimiento. Considerando estos resultados, es de esperar que próximamente se realicen ensayos clínicos fase 2 utilizando estos tipos celulares. Actualmente, se encuentran en desarrollo dos ensayos clínicos fase 1, que buscan determinar la seguridad y eficacia de la infusión intracoronaria de CSC alogénicas, en pacientes con cardiomiopatía isquémica (NCT01458405, NCT01496209).

El proceso de remodelamiento miocárdico comienza tempranamente post IAM, por lo que una infusión temprana de células madre pudiera tener un mayor impacto en frenar este proceso patológico. Esta interrogante ha sido evaluada por dos ensayos clínicos controlados, que estudiaron la respuesta a la terapia celular administrada en cortes temporales distintos. El SWISS-AMI $(n=192)$ pretendía determinar si existían diferencias entre la infusión intracoronaria temprana (5-7 días) versus tardía (3-4 semanas) de BMMC en pacientes post IAM sometidos a terapia de reperfusión ${ }^{61}$. Por otra parte, el estudio TIME $(\mathrm{n}=120)$ evaluó el impacto sobre la función ventricular de la administración de BMMC al tercer y séptimo día post angioplastía, en pacientes post $\mathrm{IAM}^{62}$. Estos estudios no reportaron con este tipo celular, diferencias entre el tratamiento precoz y tardío, sobre la función ventricular.

Los principales estudios de terapia celular en IAM se resumen en la Tabla 1.

\section{Terapia celular en cardiopatías crónicas}

Ensayos recientes han demostrado algunas mejoras clínicas y de función ventricular en pacientes con miocardiopatías de distintas etiologías. Por ejemplo, el estudio STAR-heart publicó su experiencia con el trasplante intracoronario de BMMC en pacientes con cardiopatía isquémica crónica con FEVI $<35 \%$ con seguimiento prolongado ${ }^{63}$. La terapia celular mejoró la función ventricular, la calidad de vida y la sobrevida, sugiriendo que la terapia puede ser eficaz aun a distancia de un evento coronario agudo ${ }^{63}$. Un reciente metaanálisis considerando otros 10 estudios aleatorios en pacientes con cardiopatía isquémica $(n=519)$, reportó que el tratamiento con BMMC se asoció a una mejoría significativa de la FEVI $(+4,48 \%$; $\mathrm{p}=0,0001)$, menor volumen de fin de sístole $(-20,64 \mathrm{ml}, \mathrm{p}=0,001)$, y menor volumen de fin de diástole $(-16,71 \mathrm{ml} ; \mathrm{p}=0,03)^{64}$. Experiencias algo contradictorias, como el DanCell-CHF, no detectaron un efecto significativo sobre la función ventricular, incluso con inyecciones repetidas de BMMC autólogas, pero si una mejoría clínica en pacientes con cardiopatía isquémica ${ }^{65}$. En cuanto al uso de MSC para el tratamiento de esta patología, recientemente el estudio POSEIDON $(\mathrm{n}=30)$ ha señalado un efecto beneficioso de la administración intramiocárdica de MSC sobre la capacidad funcional y el remodelamiento ventricular a 1 año de seguimiento ${ }^{66}$. Este estudio tiene la particularidad de comparar el tratamiento con células autólogas versus células alogénicas, demostrando la seguridad en la administración de ambas $^{66}$. El pequeño tamaño muestral y la falta de un grupo control, hacen cuestionable algunas de sus conclusiones sobre eficacia. Se encuentra en 
Tabla 1. Efecto terapéutico de las terapias celulares post infarto agudo al miocardio

\begin{tabular}{|c|c|c|c|c|c|c|c|}
\hline Ensayo (Cita) & $n(t)$ & Tratamiento & Vía & n células & $\mathbf{S}$ & Resultados & Imagen \\
\hline $\begin{array}{l}\text { TOPCARE-AMI } \\
(40)\end{array}$ & $\begin{array}{c}31 \\
(16)\end{array}$ & $\begin{array}{l}\text { BMMC vs } \\
\text { Control }\end{array}$ & IC & $1,6 \times 10^{7}$ & 60 & $\begin{array}{l}\text { Procedimiento seguro } \\
\uparrow \text { FEVI: }+12,3 \pm 7,7 \% \text { al } 5^{\circ} \text { año }(p<0,001) \\
\text { LVEDV sin cambios significativos } \\
\downarrow \text { LVESV: }-4,4 \pm 6,3 \text { al } 5^{\circ} \text { año }(p<0,02) \\
\uparrow \text { Tamaño de infarto }(p<0,001) \\
\downarrow \text { Contractilidad regional }\end{array}$ & RNM \\
\hline $\begin{array}{l}\text { BALANCE } \\
(46)\end{array}$ & $\begin{array}{l}124 \\
(62)\end{array}$ & $\begin{array}{l}\text { BMMC vs } \\
\text { Control }\end{array}$ & IC & $6,1 \times 10^{7}$ & 60 & 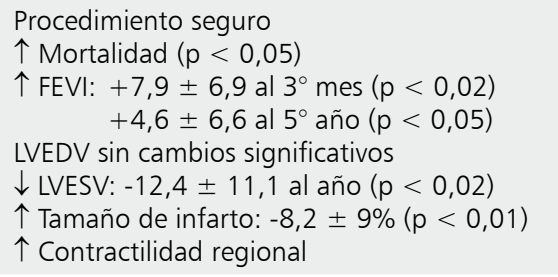 & VG \\
\hline $\begin{array}{l}\text { STIM } \\
(50)\end{array}$ & $\begin{array}{c}42 \\
(23)\end{array}$ & $\begin{array}{l}\text { BMMC vs } \\
\text { Control }\end{array}$ & IC & $15,8 \times 10^{7}$ & 60 & $\begin{array}{l}\text { Procedimiento seguro } \\
\uparrow \text { FEVI: }+8,5 \% \text { al } 5^{\circ} \text { año }(p<0,04) \\
\text { LVEDV sin cambios significativos } \\
\text { LVESV sin cambios significativos }\end{array}$ & ECO \\
\hline $\begin{array}{l}\text { BOOST } \\
(54)\end{array}$ & $\begin{array}{c}60 \\
(30)\end{array}$ & $\begin{array}{l}\text { BMMC vs } \\
\text { Control }\end{array}$ & IC & $9,5 \times 10^{6}$ & 60 & $\begin{array}{l}\text { Procedimiento seguro } \\
\uparrow \text { FEVI: }+6,7 \pm 6,5 \% \text { al } 6^{\circ} \text { mes ( } p<0,01 \text { ) } \\
\text { Luego sin diferencias respecto a basal } \\
\text { LVEDV sin cambios significativos vs control } \\
\text { LVESV sin cambios significativos vs control } \\
\uparrow \text { Contractilidad regional }\end{array}$ & $\mathrm{RMC}$ \\
\hline $\begin{array}{l}\text { ASTAMI } \\
(45)\end{array}$ & $\begin{array}{l}100 \\
(50)\end{array}$ & $\begin{array}{l}\text { BMMC vs } \\
\text { Control }\end{array}$ & IC & $6,8 \times 10^{7}$ & 36 & $\begin{array}{l}\text { Procedimiento seguro } \\
\text { FEVI sin cambios significativos } \\
\text { LVEDV sin cambios significativos } \\
\text { LVESV sin cambios significativos } \\
\uparrow \text { Contractilidad regional }\end{array}$ & ECO \\
\hline $\begin{array}{l}\text { REPAIR-AMI } \\
(47,48)\end{array}$ & $\begin{array}{c}59 \\
(26)\end{array}$ & $\begin{array}{l}\text { BMMC vs } \\
\text { Placebo }\end{array}$ & IC & $23 \times 10^{7}$ & 24 & $\begin{array}{l}\text { Procedimiento seguro } \\
\downarrow \text { Mortalidad }(p<0,05) \\
\uparrow \text { FEVI: }+6,5 \pm 2,4 \% \text { al } 2^{\circ} \text { año }(p<0,01) \\
\text { LVEDV sin cambios significativos } \\
\text { LVESV sin cambios significativos } \\
\uparrow \text { Contractilidad regional }\end{array}$ & RMC \\
\hline $\begin{array}{l}\text { PROCHYMAL } \\
(58)\end{array}$ & $\begin{array}{c}34 \\
(20)\end{array}$ & MSC vs Placebo & EV & $\leq 5 \times 10^{6}$ & 12 & $\begin{array}{l}\text { Procedimiento seguro } \\
\uparrow \text { FEVI: }+5,2 \pm 1,9 \% \text { al año }(p=0,005) \\
\text { LVEDV sin cambios significativos } \\
\text { LVESV sin cambios significativos }\end{array}$ & RMC \\
\hline $\begin{array}{l}\text { SCIPIO } \\
(59)\end{array}$ & $\begin{array}{c}23 \\
(16)\end{array}$ & CSC vs Control & IC & $1 \times 10^{6}$ & 12 & $\begin{array}{l}\text { Procedimiento seguro } \\
\uparrow \text { FEVI: }+12,3 \pm 2,1 \%(p<0,001) \text {. Sólo } 8 \\
\text { pacientes infundidos completaron el estudio } \\
\downarrow \text { Tamaño de infarto: } 30 \%(p<0,04)\end{array}$ & $\mathrm{RMC}$ \\
\hline $\begin{array}{l}\text { CADUCEUS } \\
(60)\end{array}$ & $\begin{array}{c}25 \\
(17)\end{array}$ & CDC vs Control & IC & $2,16 \times 10^{7}$ & 12 & $\begin{array}{l}\text { Procedimiento seguro } \\
\text { FEVI sin cambios significativos } \\
\text { LVEDV sin cambios significativos } \\
\text { LVESV sin cambios significativos } \\
\downarrow \text { Tamaño de infarto: } 42 \%(p<0,001) \\
\uparrow \text { Contractilidad regional }\end{array}$ & RMC \\
\hline
\end{tabular}

Se incluyeron ensayos clínicos con mayor número de pacientes y seguimiento por tipo celular. Los cambios están expresados en relación a la situación basal según lo reportado en la literatura. n: Número de pacientes, (t): número de pacientes tratados. S: Tiempo de seguimiento. BMMC: células mononucleares de médula ósea. MSC: Células madre mesenquimales. CSC: Células madre cardiacas. CDC: Cardioesferas. FEVI: Fracción de eyección del ventrículo izquierdo. LVESV: Volumen de fin de sístole. LVEDV: Volumen de fin de diástole. ECO: Ecocardiografía. RMC: Resonancia magnética cardiaca. VG: Ventriculografía. 
desarrollo el ensayo clínico aleatorizado TAC-HFT que comparará la seguridad y eficacia de la administración intracardiaca de BMMC versus MSC en pacientes con cardiopatía isquémica crónica ${ }^{67}$.

En pacientes con miocardiopatía de etiología no isquémica, existe una experiencia más reducida $^{68-75}$. El ensayo aleatorizado ABCD señaló un aumento significativo y persistente de la FEVI y mejora en la calidad de vida en pacientes con cardiopatía dilatada, tratados con BMMC autólogas en su seguimiento a 3 años ${ }^{72}$. Otro estudio aleatorizado recientemente publicado, también reveló un aumento persistente de la FEVI, así como una reducción significativa de la morbimortalidad en pacientes tratados con células BMMC autólogas, a 60 meses $^{74}$. En nuestro ensayo clínico controlado en pacientes con cardiopatía dilatada, también se evidenció una mejoría significativa y persistente de la capacidad funcional, documentándose adicionalmente un aumento de la FEVI a partir de los 6 meses de tratamiento $(26,75 \pm 4,85$ a 37,82 $\pm 6,97 \%, p=0,001)$, en los pacientes infundidos con $\mathrm{BMMC}^{75}$.

Los principales estudios de terapia celular en cardiopatías de diversa etiología se resumen en la Tabla 2.

Tabla 2. Efecto terapéutico de las terapias celulares en cardiopatías crónicas de diferente etiología

\begin{tabular}{|c|c|c|c|c|c|c|c|}
\hline Ensayo (Cita) & $n(t)$ & Tratamiento & Vía & $\mathbf{n}$ células & $\mathbf{S}$ & Resultados & Imagen \\
\hline $\begin{array}{l}\text { STAR-heart } \\
(63)\end{array}$ & $\begin{array}{l}391 \\
(191)\end{array}$ & $\begin{array}{l}\text { BMMC vs } \\
\text { control }\end{array}$ & IC & $6,6 \times 10^{7}$ & 60 & $\begin{array}{l}\text { Procedimiento seguro } \\
\uparrow \text { FEVI: }+6,2 \pm 8,4 \text { al } 5^{\circ} \text { año }(p<0,02) \\
\downarrow \text { LVEDV : }-9 \pm 27,4 \text { al } 5^{\circ} \text { año }(p<0,02) \\
\downarrow \text { LVESV: }-14,4 \pm 27 \text { al } 5^{\circ} \text { año }(p<0,02) \\
\downarrow \text { Tamaño de infarto: }-11,4 \%(p<0,05) \\
\uparrow \text { Contractilidad regional }\end{array}$ & ECO \\
\hline $\begin{array}{l}\text { FOCUS-CCTRN } \\
(80)\end{array}$ & $\begin{array}{c}92 \\
(61)\end{array}$ & $\begin{array}{l}\text { BMMC vs } \\
\text { control }\end{array}$ & $\mathrm{IM}$ & $10 \times 10^{7}$ & 6 & $\begin{array}{l}\text { Procedimiento seguro } \\
\uparrow \text { FEVI: }+1,4 \pm 5,2(p<0,03) \\
\text { LVESV sin cambios significativos } \\
\text { Tamaño de infarto sin cambios significativos } \\
\text { Contractilidad regional sin cambios }\end{array}$ & $\begin{array}{l}\text { ECO, } \\
\text { SPECT }\end{array}$ \\
\hline $\begin{array}{l}\text { DanCell-CHF } \\
\text { (65) }\end{array}$ & $\begin{array}{c}32 \\
(32)\end{array}$ & BMMC & IC & $\begin{array}{c}9,7 \times 10^{6} \\
+ \\
14,1 \times 10^{6}\end{array}$ & 12 & $\begin{array}{l}\text { Procedimiento seguro } \\
\text { Mejoría NYHA ( } p<0,0001 \text { ) } \\
\text { Mejoría test de esfuerzo hasta los } 8 \text { meses } \\
\text { FEVI sin cambios significativos } \\
\text { LVESV sin cambios significativos } \\
\text { Contractilidad regional sin cambios }\end{array}$ & ECO \\
\hline $\begin{array}{l}\text { POSEIDON } \\
\text { (66) }\end{array}$ & $\begin{array}{c}30 \\
(30)\end{array}$ & $\begin{array}{l}\text { MSC Autólogas } \\
\text { vs Alogénicas }\end{array}$ & $\mathrm{IM}$ & $2-20 \times 10^{7}$ & 12 & $\begin{array}{l}\text { Procedimiento seguro } \\
\downarrow \text { Tamaño de infarto: }-33,21 \%(p<0,001) \\
\text { FEVI sin cambios significativos } \\
\text { LVEDV sin cambios significativos } \\
\text { LVESV sin cambios significativos } \\
\text { Mejoría en encuestasQoL }(p<0,05)\end{array}$ & $\mathrm{TC}$ \\
\hline $\begin{array}{l}A B C D \\
(72)\end{array}$ & $\begin{array}{l}81 \\
(41)\end{array}$ & $\begin{array}{l}\text { BMMC vs } \\
\text { control }\end{array}$ & IC & $2,7 \times 10^{6}$ & 36 & $\begin{array}{l}\text { Procedimiento seguro } \\
\uparrow \text { FEVI: } 22,5 \pm 8,3 \text { a } 28,4 \pm 11,8 \%(p<0,05) \\
\text { LVEDV sin cambios significativos } \\
\downarrow \text { LVESV: } 137 \pm 63 \text { a } 120 \pm 52(p<0,05) \\
\text { Mejoría en encuestasQoL }(p<0,05)\end{array}$ & ECO \\
\hline $\begin{array}{l}\text { Vrtovec et al } \\
(74)\end{array}$ & $\begin{array}{l}110 \\
(55)\end{array}$ & $\begin{array}{l}\text { BMMC vs } \\
\text { control }\end{array}$ & IC & $11 \times 10^{7}$ & 60 & $\begin{array}{l}\text { Procedimiento seguro } \\
\downarrow \text { Mortalidad }(p=0,01) \\
\uparrow \text { FEVI: }+5,7 \%(p<0,05) \\
\text { LVEDV sin cambios significativos } \\
\downarrow \text { NT-proBNP }(p<0,001)\end{array}$ & ECO \\
\hline
\end{tabular}

Se incluyeron ensayos clínicos con mayor número de pacientes y seguimiento por tipo celular. Los cambios están expresados en relación a la situación basal según lo reportado en la literatura. n: Número de pacientes, (t): número de pacientes tratados. S: Tiempo de seguimiento. BMMC: células mononucleares de medula ósea. MSC: Células madre mesenquimales. FEVI: Fracción de eyección del ventrículo izquierdo. LVESV: Volumen de fin de sístole. LVEDV: Volumen de fin de diástole. QoL: Calidad de Vida. ECO: Ecocardiografía. TC: Tomografía computada. 


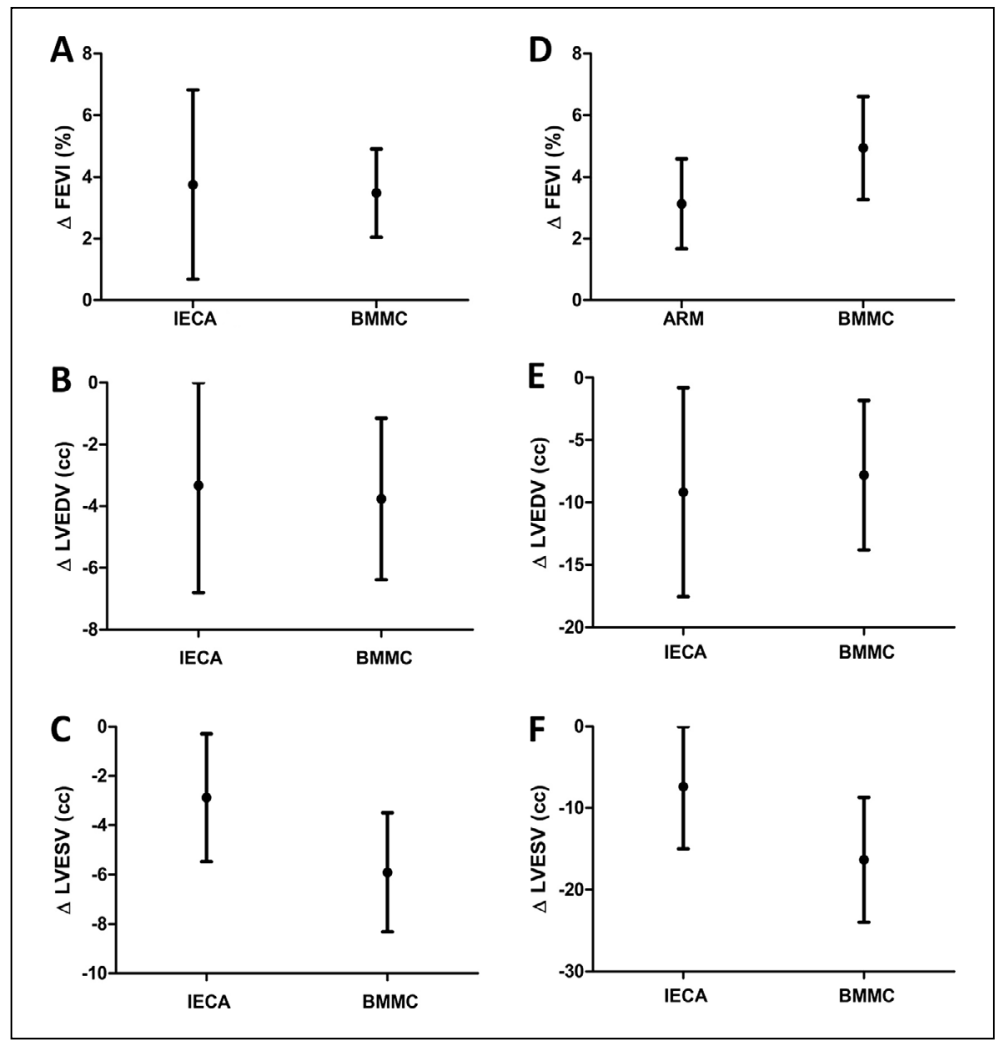

Figura 2. Impacto sobre parámetros de función ventricular de la terapia celular versus bloqueo del sistema renina angiotensina aldosterona. Se señala el efecto de la terapia con inhibidores de la enzima convertidora de angiotensina (IECA), antagonistas del receptor de mineral o corticoides (ARM) o células mononucleares de médula ósea (BMMC) sobre la de fracción de eyección ventricular izquierda (FEVI), volumen de fin de diástole (LVEDV) y volumen de fin de sístole (LVESV) en pacientes post IAM (A, B, C) y pacientes con cardiopatía isquémica crónica $(\mathbf{D}, \mathbf{E}, \mathbf{F})$. Los valores graficados corresponden a las media y su respectivo intervalo de confianza (IC 95\%) reportado por meta-análisis y/o revisiones sistemáticas de las distintas terapias considerando estudios con 6 meses a 1 año de seguimiento ${ }^{36,81,82}$.

\section{Conclusión}

La evidencia disponible es consistente en demostrar la seguridad del tratamiento con células madre en pacientes cardiópatas. Los meta-análisis recientes basados en ensayos clínicos fase 1-2 sugieren un beneficio significativo sobre la morbimortalidad en pacientes post IAM o cardiopatías crónicas de diferente etiología, en pacientes sometidos a terapia celular, beneficio que deberá ser confirmado por ensayos clínicos en fase 3 , actualmente en desarrollo (NCT01569178). Adicionalmente, estos estudios han reportado beneficios sobre la función ventricular de pacientes tratados, similares a los demostrados por otras terapias modificadoras del remodelamiento miocárdico incorporadas en las guías clínicas actuales (Figura 2). No obstante lo anterior, esta eficacia ha sido parcialmente cuestionada por estudios que no encontraron cambios en la FEVI, variable dependiente de cambios de la precarga, la postcarga, la contractilidad miocárdica y la activación neurohu- moral entre otras ${ }^{76}$. Variables como la disminución del tamaño de infarto, dimensiones ventriculares y/o la mejoría de la función miocárdica regional, que pueden tener escaso impacto sobre la FEVI, han sido propuestas como mejores marcadores de eficacia de esta terapia ${ }^{76}$. Siguiendo esta idea, estudios más recientes o en desarrollo han privilegiado la resonancia magnética cardiaca, como mejor método para evaluar remodelamiento miocárdico, por sobre otros estudios imagenológicos.

Es necesario resaltar que la terapia celular es complementaria al tratamiento farmacológico convencional, habiéndose descrito su asociación sinérgica en estudios experimentales ${ }^{77-79}$. Finalmente, es importante mencionar que aunque la terapia celular representa un avance promisorio para la cardiología, aún quedan muchas dudas por resolver, incluyendo los criterios de selección de pacientes proclives a esta modalidad de tratamiento, las fuentes celulares a utilizar, vías de implante, cantidad de células a implantar y momento de su aplicación, entre otros. 


\section{Referencias}

1. Rosamond W, Flegal K, Friday G, Furie K, Go A, Greenlund K, et al. Heart disease and stroke statistics-2007 update: a report from the American Heart Association Statistics Committee and Stroke Statistics Subcommittee. Circulation 2007; 115 (5): e69-171.

2. Pearson TA, Blair SN, Daniels SR, Eckel RH, Fair JM, Fortmann SP, et al. AHA Guidelines for Primary Prevention of Cardiovascular Disease and Stroke: 2002 Update: Consensus Panel Guide to Comprehensive Risk Reduction for Adult Patients Without Coronary or Other Atherosclerotic Vascular Diseases. American Heart Association Science Advisory and Coordinating Committee. Circulation 2002; 106 (3): 388-91.

3. Vigen R, Maddox TM, Allen LA. Aging of the United States population: impact on heart failure. Curr Heart Fail Rep 2012; 9 (4): 369-74.

4. Monti M, Perotti C, Del Fante C, Cervio M, Redi CA. Stem cells: sources and therapies. Biol Res 2012; 45 (3): 207-14.

5. Orlic D, Kajstura J, Chimenti S, Bodine DM, Leri A, Anversa P. Transplanted adult bone marrow cells repair myocardial infarcts in mice. Ann N Y Acad Sci 2001; 938: 221-9.

6. Fuchs S, Baffour R, Zhou YF, Shou M, Pierre A, Tio FO, et al. Transendocardial delivery of autologous bone marrow enhances collateral perfusion and regional function in pigs with chronic experimental myocardial ischemia. J Am Coll Cardiol 2001; 37 (6): 1726-32.

7. Strauer BE, Brehm M, Zeus T, Kostering M, Hernández A, Sorg RV, et al. Repair of infarcted myocardium by autologous intracoronary mononuclear bone marrow cell transplantation in humans. Circulation 2002; 106 (15): 1913-8.

8. Assmus B, Schachinger V, Teupe C, Britten M, Lehmann R, Dobert N, et al. Transplantation of Progenitor Cells and Regeneration Enhancement in Acute Myocardial Infarction (TOPCARE-AMI). Circulation 2002; 106 (24): 3009-17.

9. Mauritz C, Martens A, Rojas SV, Schnick T, Rathert C, Schecker N, et al. Induced pluripotent stem cell (iPSC)derived Flk-1 progenitor cells engraft, differentiate, and improve heart function in a mouse model of acute myocardial infarction. Eur Heart J 2011; 32 (21): 263441.

10. Mandel Y, Weissman A, Schick R, Barad L, Novak A, Meiry G, et al. Human embryonic and induced pluripotent stem cell-derived cardiomyocytes exhibit beat rate variability and power-law behavior. Circulation 2012; 125 (7): 883-93.
11. Yu T, Miyagawa S, Miki K, Saito A, Fukushima S, Higuchi $\mathrm{T}$, et al. In vivo differentiation of induced pluripotent stem cell-derived cardiomyocytes. Circ J 2013; 77 (5): 1297-306.

12. Zhang Y, Wang D, Chen M, Yang B, Zhang F, Cao K. Intramyocardial transplantation of undifferentiated rat induced pluripotent stem cells causes tumorigenesis in the heart. PLoS One 2011; 6 (4): e19012.

13. Diez Villanueva P, Sanz-Ruiz R, Núñez García A, Fernández Santos ME, Sánchez PL, Fernández-Aviles F. Functional multipotency of stem cells: what do we need from them in the heart? Stem Cells Int 2012; 2012 : 817364.

14. Strauer BE, Steinhoff G. 10 years of intracoronary and intramyocardial bone marrow stem cell therapy of the heart: from the methodological origin to clinical practice. J Am Coll Cardiol 2011; 58 (11): 1095-104.

15. Gaebel R, Furlani D, Sorg H, Polchow B, Frank J, Bieback $\mathrm{K}$, et al. Cell origin of human mesenchymal stem cells determines a different healing performance in cardiac regeneration. PLoS One 2011; 6 (2): e15652.

16. Williams AR, Hare JM. Mesenchymal stem cells: biology, pathophysiology, translational findings, and therapeutic implications for cardiac disease. Circ Res 2011; 109 (8): 923-40.

17. Dimmeler S, Leri A. Aging and disease as modifiers of efficacy of cell therapy. Circ Res 2008; 102 (11): 131930.

18. Beltrami AP, Barlucchi L, Torella D, Baker M, Limana F, Chimenti S, et al. Adult cardiac stem cells are multipotent and support myocardial regeneration. Cell 2003; 114 (6): 763-76.

19. Davis DR, Ruckdeschel Smith R, Marban E. Human cardiospheres are a source of stem cells with cardiomyogenic potential. Stem Cells 2010; 28 (5): 903-4.

20. Leri A, Kajstura J, Anversa P. Role of cardiac stem cells in cardiac pathophysiology: a paradigm shift in human myocardial biology. Circ Res 2011; 109 (8): 941-61.

21. Smith RR, Barile L, Cho HC, Leppo MK, Hare JM, Messina E, et al. Regenerative potential of cardiosphere-derived cells expanded from percutaneous endomyocardial biopsy specimens. Circulation 2007; 115 (7): 896-908.

22. Chimenti I, Smith RR, Li TS, Gerstenblith G, Messina E, Giacomello A, et al. Relative roles of direct regeneration versus paracrine effects of human cardiosphere-derived cells transplanted into infarcted mice. Circ Res 2010; 106 (5): 971-80.

23. Toma C, Pittenger MF, Cahill KS, Byrne BJ, Kessler PD. Human mesenchymal stem cells differentiate to a cardiomyocyte phenotype in the adult murine heart. Circulation 2002; 105 (1): 93-8. 
Terapia con células madre en cardiopatías - J. Bartolucci et al

24. Nagaya N, Kangawa $K$, Itoh $T$, Iwase $T$, Murakami S, Miyahara Y, et al. Transplantation of mesenchymal stem cells improves cardiac function in a rat model of dilated cardiomyopathy. Circulation 2005; 112 (8): 1128-35.

25. Tang J, Xie Q, Pan G, Wang J, Wang M. Mesenchymal stem cells participate in angiogenesis and improve heart function in rat model of myocardial ischemia with reperfusion. Eur J Cardiothorac Surg 2006; 30 (2): 353-61.

26. Nygren JM, Jovinge S, Bereitbach M, Säwen P, Röll W, Hescheler J, et al. Bone marrow-derived hematopoietic cells generate cardiomyocytes at a low frequency through cell fusion, but not transdifferentiation. Nat Med 2004; 10 (5): 494-501.

27. Oh H, Bradfute SB, Gallardo TD, Nakamura T, Gaussin V, Mishina Y, et al. Cardiac progenitor cells from adult myocardium: homing, differentiation, and fusion after infarction. Proc Natl Acad Sci U S A 2003; 100 (21): 12313-8.

28. Gnecchi M, Zhang Z, Ni A, Dzau VJ. Paracrine mechanisms in adult stem cell signaling and therapy. Circ Res 2008; 103 (11): 1204-19.

29. Berry MF, Engler AJ, Woo YJ, Pirolli TJ, Bish LT, Jayasankar V, et al. Mesenchymal stem cell injection after myocardial infarction improves myocardial compliance. Am J Physiol Heart Circ Physiol 2006; 290 (6): H2196203.

30. Hatzistergos KE, Quevedo H, Oskouei BN, Hu Q, Feigenbaum GS, Margitich IS, et al. Bone marrow mesenchymal stem cells stimulate cardiac stem cell proliferation and differentiation. Circ Res 2010; 107 (7): 913-22.

31. Crisostomo PR, Abarbanell AM, Wang M, Lahm T, Wang Y, Meldrum DR. Embryonic stem cells attenuate myocardial dysfunction and inflammation after surgical global ischemia via paracrine actions. Am J Physiol Heart Circ Physiol 2008; 295 (4): H1726-35.

32. Molina EJ, Palma J, Gupta D, Torres D, Gaughan JP, Houser $S$, et al. Improvement in hemodynamic performance, exercise capacity, inflammatory profile, and left ventricular reverse remodeling after intracoronary delivery of mesenchymal stem cells in an experimental model of pressure overload hypertrophy. J Thorac Cardiovasc Surg 2008; 135 (2): 292-9, 9 e1.

33. Torre-Amione G. Immune activation in chronic heart failure. Am J Cardiol 2005; 95 (11A): 3C-8C; discussion 38C-40C.

34. Ohtsuka T, Hamada M, Inoue K, Ohshima K, Sujzuki J, Matsunaka T, et al. Relation of circulating interleukin-6 to left ventricular remodeling in patients with reperfused anterior myocardial infarction. Clin Cardiol 2004; 27 (7): 417-20.
35. Kang HJ, Kim HS, Zhang SY, Park KW, Cho HJ, Koo $\mathrm{BK}$, et al. Effects of intracoronary infusion of peripheral blood stem-cells mobilised with granulocyte-colony stimulating factor on left ventricular systolic function and restenosis after coronary stenting in myocardial infarction: the MAGIC cell randomised clinical trial. Lancet 2004; 363 (9411): 751-6.

36. Jeevanantham V, Butler M, Saad A, Abdel-Latif A, ZubaSurma EK, Dawn B. Adult bone marrow cell therapy improves survival and induces long-term improvement in cardiac parameters: a systematic review and metaanalysis. Circulation 2012; 126 (5): 551-68.

37. van der Spoel TI, Jansen of Lorkeers SJ, Agostoni P, van Belle E, Gyongyosi M, Sluijter JP, et al. Human relevance of pre-clinical studies in stem cell therapy: systematic review and meta-analysis of large animal models of ischaemic heart disease. Cardiovasc Res 2011; 91 (4): 649-58.

38. Chen SL, Fang WW, Ye F, Liu YH, Qian J, Shan SJ, et al. Effect on left ventricular function of intracoronary transplantation of autologous bone marrow mesenchymal stem cell in patients with acute myocardial infarction. Am J Cardiol 2004; 94 (1): 92-5.

39. Schachinger V, Assmus B, Britten MB, Honold J, Lehmann R, Teupe C, et al. Transplantation of progenitor cells and regeneration enhancement in acute myocardial infarction: final one-year results of the TOPCARE-AMI Trial. J Am Coll Cardiol 2004; 44 (8): 1690-9.

40. Leistner DM, Fischer-Rasokat U, Honold J, Seeger FH, Schachinger V, Lehmann R, et al. Transplantation of progenitor cells and regeneration enhancement in acute myocardial infarction (TOPCARE-AMI): final 5-year results suggest long-term safety and efficacy. Clin Res Cardiol 2011; 100 (10): 925-34.

41. Schachinger V, Erbs S, Elsasser A, Haberbosch W, Hambrecht $\mathrm{R}$, Holschermann $\mathrm{H}$, et al. Improved clinical outcome after intracoronary administration of bonemarrow-derived progenitor cells in acute myocardial infarction: final 1-year results of the REPAIR-AMI trial. Eur Heart J 2006; 27 (23): 2775-83.

42. Janssens S, Dubois C, Bogaert J, Theunissen K, Deroose C, Desmet W, et al. Autologous bone marrow-derived stem-cell transfer in patients with ST-segment elevation myocardial infarction: double-blind, randomised controlled trial. Lancet 2006; 367 (9505): 113-21.

43. Lunde K, Solheim S, Aakhus S, Arnesen H, Abdelnoor M, Egeland T, et al. Intracoronary injection of mononuclear bone marrow cells in acute myocardial infarction. $\mathrm{N}$ Engl J Med 2006; 355 (12): 1199-209.

44. Lunde K, Solheim S, Aakhus S, Arnesen H, Moum T, Abdelnoor M, et al. Exercise capacity and quality of life 
after intracoronary injection of autologous mononuclear bone marrow cells in acute myocardial infarction: Results from the Autologous Stem cell Transplantation in Acute Myocardial Infarction (ASTAMI) randomized controlled trial. Am Heart J 2007; 154 (710): 710e1-e8.

45. Beitnes JO, Gjesdal O, Lunde K, Solheim S, Edvardsen $\mathrm{T}$, Arnesen $\mathrm{H}$, et al. Left ventricular systolic and diastolic function improve after acutemyocardial infarction treated with acute percutaneous coronary intervention, but are not influenced by intracoronary injection of autologous mononuclear bone marrow cells: a 3 year serial echocardiographic sub-study of the randomizedcontrolled ASTAMI study. Eur J Echocardiogr 2011; 12: 98-106.

46. Yousef M, Schannwell CM, Kostering M, Zeus T, Brehm M, Strauer BE. The BALANCE Study: clinical benefit and long-term outcome after intracoronary autologous bone marrow cell transplantation in patients with acute myocardial infarction. J Am Coll Cardiol 2009; 53 (24): 2262-9.

47. Assmus B, Rolf A, Erbs S, Elsasser A, Haberbosch W, Hambrecht R, et al. Clinical outcome 2 years after intracoronary administration of bone marrow-derived progenitor cells in acute myocardial infarction. Circ Heart Fail 2010; 3 (1): 89-96.

48. Rolf A, Assmus B, Schachinger V, Rixe J, Mollmann S, Mollmann $\mathrm{H}$, et al. Maladaptive hypertrophy after acute myocardial infarction positive effect of bone marrowderived stem cell therapy on regional remodeling measured by cardiac MRI. Clin Res Cardiol 2011; 100 (11): 983-92.

49. Traverse JH, McKenna DH, Harvey K, Jorgenso BC, Olson RE, Bostrom N, et al. Results of a phase 1, randomized, double-blind, placebo-controlled trial of bone marrow mononuclear stem cell administration in patients following ST-elevation myocardial infarction. Am Heart J 2010; 160 (3): 428-34.

50. Moccetti T, Surder D, Klersy C, Vassalli G, Crljenica C, Rossi MG, et al. Sustained improvement in left ventricular function after bone marrow derived cell therapy in patients with acute ST elevation myocardial infarction. A 5-year follow-up from the Stem Cell Transplantation in Ischaemic Myocardium Study. Swiss Med Wkly 2012; 142: w13632.

51. Quyyumi AA, Waller EK, Murrow J, Esteves F, Galt J, Oshinski J, et al. CD34(+) cell infusion after ST elevation myocardial infarction is associated with improved perfusion and is dose dependent. Am Heart J 2011; 161 (1): 98-105.

52. Meluzin J, Janousek S, Mayer J, Groch L, Hornacek I, Hlinomaz O, et al. Three-, 6-, and 12-month results of autologous transplantation of mononuclear bone marrow cells in patients with acute myocardial infarction. Int J Cardiol 2008; 128 (2): 185-92.

53. Wollert KC, Meyer GP, Lotz J, Ringes-Lichtenberg S, Lippolt $\mathrm{P}$, Breidenbach $\mathrm{C}$, et al. Intracoronary autologous bone-marrow cell transfer after myocardial infarction: the BOOST randomised controlled clinical trial. Lancet 2004; 364 (9429): 141-8.

54. Meyer GP, Wollert KC, Lotz J, Pirr J, Rager U, Lippolt $\mathrm{P}$, et al. Intracoronary bone marrow cell transfer after myocardial infarction: 5-year follow-up from the randomized-controlled BOOST trial. Eur Heart J 2009; 30 (24): 2978-84.

55. Meyer GP, Wollert KC, Lotz J, Steffens J, Lippolt P, Fichtner S, et al. Intracoronary bone marrow cell transfer after myocardial infarction: eighteen months' follow-up data from the randomized, controlled BOOST (BOne marrOw transfer to enhance ST-elevation infarct regeneration) trial. Circulation 2006; 113 (10): 1287-94.

56. Katritsis DG, Sotiropoulou PA, Karvouni E, Karabinos I, Korovesis S, Pérez SA, et al. Transcoronary transplantation of autologous mesenchymal stem cells and endothelial progenitors into infarcted human myocardium. Catheter Cardiovasc Interv 2005; 65 (3): 321-9.

57. Katritsis DG, Sotiropoulou P, Giazitzoglou E, Karvouni E, Papamichail M. Electrophysiological effects of intracoronary transplantation of autologous mesenchymal and endothelial progenitor cells. Europace 2007; 9 (3): 167-71.

58. Hare JM, Traverse JH, Henry TD, Dib N, Strumpf RK, Schulman SP, et al. A randomized, double-blind, placebo-controlled, dose-escalation study of intravenous adult human mesenchymal stem cells (prochymal) after acute myocardial infarction. J Am Coll Cardiol 2009; 54 (24): 2277-86.

59. Bolli R, Chugh AR, D'Amario D, Loughran JH, Stoddard MF, Ikram S, et al. Cardiac stem cells in patients with ischaemic cardiomyopathy (SCIPIO): initial results of a randomised phase 1 trial. Lancet 2011; 378 (9806): 1847-57.

60. Makkar RR, Smith RR, Cheng K, Malliaras K, Thomson LE, Berman D, et al. Intracoronary cardiosphere-derived cells for heart regeneration after myocardial infarction (CADUCEUS): a prospective, randomised phase 1 trial. Lancet 2012; 379 (9819): 895-904.

61. Surder D, Schwitter J, Moccetti T, Astori G, Rufibach K, Plein $\mathrm{S}$, et al. Cell-based therapy for myocardial repair in patients with acute myocardial infarction: rationale and study design of the SWiss multicenter Intracoronary Stem cells Study in Acute Myocardial Infarction (SWISSAMI). Am Heart J 2010; 160 (1): 58-64. 
62. Traverse JH, Henry TD, Pepine CJ, Willerson J, Zhao DX, Ellis SG, et al. Effect of the use and timing of bone marrow mononuclear cell delivery on left ventricular function after acute myocardial infarction: The time randomized trial. JAMA 2012; 308 (22): 2380-9.

63. Strauer BE, Yousef M, Schannwell CM. The acute and long-term effects of intracoronary Stem cell Transplantation in 191 patients with chronic heARt failure: the STAR-heart study. Eur J Heart Fail 2010; 12 (7): 721-9.

64. Kandala J, Upadhyay GA, Pokushalov E, Wu S, Drachman DE, Singh JP. Meta-Analysis of Stem Cell Therapy in Chronic Ischemic Cardiomyopathy. Am J Cardiol 2013 (en prensa)

65. Diederichsen AC, Moller JE, Thayssen P, Junker AB, Videbaek L, Saekmose SG, et al. Effect of repeated intracoronary injection of bone marrow cells in patients with ischaemic heart failure the Danish stem cell studycongestive heart failure trial (DanCell-CHF). Eur J Heart Fail 2008; 10 (7): 661-7.

66. Hare JM, Fishman JE, Gerstenblith G, DiFede Velazquez DL, Zambrano JP, Suncion VY, et al. Comparison of allogeneic vs autologous bone marrow-derived mesenchymal stem cells delivered by transendocardial injection in patients with ischemic cardiomyopathy: the POSEIDON randomized trial. JAMA 2012; 308 (22): 2369-79.

67. Trachtenberg B, Velazquez DL, Williams AR, McNiece I, Fishman J, Nguyen K, et al. Rationale and design of the Transendocardial Injection of Autologous Human Cells (bone marrow or mesenchymal) in Chronic Ischemic Left Ventricular Dysfunction and Heart Failure Secondary to Myocardial Infarction (TAC-HFT) trial: A randomized, double-blind, placebo-controlled study of safety and efficacy. Am Heart J 2011; 161 (3): 48793.

68. Seth S, Narang R, Bhargava B, Ray R, Mohanty S, Gulati $\mathrm{G}$, et al. Percutaneous intracoronary cellular cardiomyoplasty for nonischemic cardiomyopathy: clinical and histopathological results: the first-in-man $\mathrm{ABCD}$ (Autologous Bone Marrow Cells in Dilated Cardiomyopathy) trial. J Am Coll Cardiol 2006; 48 (11): 2350-1.

69. Kalil RA, Ott D, Sant'Anna R, Dias E, Marques-Pereira JP, Delgado-Canedo A, et al. Autologous transplantation of bone marrow mononuclear stem cells by minithoracotomy in dilated cardiomyopathy: technique and early results. Sao Paulo Med J 2008; 126 (2): 75-81.

70. Fischer-Rasokat U, Assmus B, Seeger FH, Honold J, Leistner D, Fichtlscherer S, et al. A pilot trial to assess potential effects of selective intracoronary bone marrow-derived progenitor cell infusion in patients with nonischemic dilated cardiomyopathy: final 1-year results of the transplantation of progenitor cells and functional regeneration enhancement pilot trial in patients with nonischemic dilated cardiomyopathy. Circ Heart Fail 2009; 2 (5): 417-23.

71. Martino HF, Oliveira PS, Souza FC, Costa PC, Assuncao ESE, Villela R, et al. A safety and feasibility study of cell therapy in dilated cardiomyopathy. Braz J Med Biol Res 2010; 43 (10): 989-95.

72. Seth S, Bhargava B, Narang R, Ray R, Mohanty S, Gulati $\mathrm{G}$, et al. The ABCD (Autologous Bone Marrow Cells in Dilated Cardiomyopathy) trial a long-term follow-up study. J Am Coll Cardiol 2010; 55 (15): 1643-4.

73. Vrtovec B, Poglajen G, Sever M, Lezaic L, Domanovic D, Cernelc P, et al. Effects of intracoronary stem cell transplantation in patients with dilated cardiomyopathy. J Card Fail 2011; 17 (4): 272-81.

74. Vrtovec B, Poglajen G, Lezaic L, Sever M, Domanovic D, Cernelc P, et al. Effects of Intracoronary CD34+ Stem Cell Transplantation in Nonischemic Dilated Cardiomyopathy Patients: 5-Year Follow-Up. Circ Res 2013; 112 (1): 165-73.

75. Bartolucci J, Carrión F, Lamich R, Pedreros P, Carvallo $\mathrm{C}$, Sanhueza P, et al. El trasplante intracoronario de células mononucleares de médula ósea mejora la función ventricular izquierda en pacientes con cardiopatía dilatada de diferente etiología. Proyecto Innova Chile $\mathrm{N}^{\circ}$ 205-4350. Rev Chil Cardiol 2013; 32: 11-20.

76. Suncion VY, Schulman IH, Hare JM. Concise review: the role of clinical trials in deciphering mechanisms of action of cardiac cell-based therapy. Stem Cells Transl Med 2012; 1 (1): 29-35.

77. Numasawa $Y$, Kimura T, Miyoshi S, Nishiyama N, Hida $\mathrm{N}$, Tsuji $\mathrm{H}$, et al. Treatment of human mesenchymal stem cells with angiotensin receptor blocker improved efficiency of cardiomyogenic transdifferentiation and improved cardiac function via angiogenesis. Stem Cells 2011; 29 (9): 1405-14.

78. Hassan F, Meduru S, Taguchi K, Kuppusamy ML, Mostafa M, Kuppusamy P, et al. Carvedilol enhances mesenchymal stem cell therapy for myocardial infarction via inhibition of caspase-3 expression. J Pharmacol Exp Ther 2012; 343 (1): 62-71.

79. Huang YL, Kuang J, Hu YZ, Song YB, Qiu RF, Mai WY. Bone marrow stromal cell transplantation combined with angiotensin-converting enzyme inhibitor treatment in rat with acute myocardial infarction and the role of insulin-like growth factor-1. Cytotherapy 2012; 14 (5): 563-9.

80. Perin EC, Willerson JT, Pepine CJ, Henry TD, Ellis SG, Zhao DX, et al. Effect of transendocardial delivery of autologous bone marrow mononuclear cells on functio- 
nal capacity, left ventricular function, and perfusion in chronic heart failure: the FOCUS-CCTRN trial. JAMA 2012; 307 (16): 1717-26.

81. Ezekowitz JA, McAlister FA. Aldosterone blockade and left ventricular dysfunction: a systematic review of randomized clinical trials. Eur Heart J 2009; 30 (4): 469-77.
82. Abdulla J, Barlera S, Latini R, Kjoller-Hansen L, Sogaard P, Christensen E, et al. A systematic review: effect of angiotensin converting enzyme inhibition on left ventricular volumes and ejection fraction in patients with a myocardial infarction and in patients with left ventricular dysfunction. Eur J Heart Fail 2007; 9 (2): 129-35. 